\title{
9. Interview mit Dr. York Langenstein, ICOM Deutschland-Präsident 2005-2009
}

\subsection{Interviewtext}

Dr. York Langenstein im Interview zu den Herausforderungen für ICOM Deutschland während seiner Amtszeit als Präsident (2005-2009), durchgeführt von Tanja Leiminger am I6. März 2020 in München:

Tanja Leininger: Die Anfänge Ihrer Mitarbeit im Vorstand von ICOM Deutschland liegen jetzt über zwei Jahrzehnte zurück, das Amt des Präsidenten haben sie vor 2005 übernommen: Was war der Ausgangspunkt für Ihr Engagement in der Verbandsarbeit des Deutschen Nationalkomitees?

Das von ICOM Deutschland initiierte Oral History-Projekt - also Zeitzeugenberichte der ehemaligen Präsidenten zur Entwicklung des Deutschen Nationalkomitees - macht mir bewusst, dass auch meine Amtszeit allmählich zu einem Kapitel unserer Verbandsgeschichte wird: Die Fragen wecken bei mir die Erinnerungen an eine sehr lebendige Zeit des internationalen Austauschs über die Rolle und die Möglichkeiten der Museen in der Gesellschaft.

Mein erster Kontakt zu ICOM Deutschland wurde über Hans-Albert Treff vermittelt, Präsident von ICOM Deutschland in den Jahren I993 bis I998 als Amtsvorgänger von Hans-Martin Hinz. In der damaligen Münchner Museumsszene stand ich als Leiter der Landesstelle für die nichtstaatlichen Museen in Bayern mit Hans-Albert Treff in einem freundschaftlich geprägten kollegialen Kontakt: Ich ließ mich von ihm als internationalem Netzwerker mit dem ihn auszeichnenden lebendigen Interesse am Blick über die Grenzen hinweg gerne einführen in die mir damals noch nicht näher bekannte Welt von ICOM. Im Kreis der gesetzten Münchner Museumsdirektoren fiel Hans-Albert Treff durch sein engagiertes Eintreten für die damals neuen Konzepte zur aktiven Einbeziehung der Besucher im Sinne der amerikanischen Science Center auf, wie er sie als Gründungsdirektor des innovativen Museums »Mensch und Natur« in den nördlichen Trakten der Anlage von Schloss Nymphenburg mit großem Erfolg umsetzte: Die dortigen Besucherzahlen ließen die meisten Kollegen vor Neid erblassen.

Wer selbst begeistert ist, kann auch andere begeistern: Hans-Albert Treff motivierte mich, sehr kurzfristig für die Neuwahl des Vorstands von ICOM Deutschland im Dezember 2004 zu kandidieren. Der Vorschlag kam tatsäch- 
lich so unvermittelt, dass ich mich bei der Mitgliederversammlung am i8. Dezember nicht einmal mehr persönlich präsentieren, sondern lediglich in einer vor der Wahl verlesenen Bewerbung vorstellen konnte. Wenn meiner Kandidatur trotzdem Erfolg beschieden war, gaben vielleicht meine Erfahrungen auf dem Feld der Verbandsarbeit der deutschen Museumsämter und Museumsverbände den Ausschlag, die ich als damaliger Leiter der Landesstelle für die nichtstaatlichen Museen in Bayern - des größten deutschen Museumsamts - einbringen konnte. Einerseits war ich mit dem Handwerk der Museumsbetreuung auf Landesebene und auch mit der Zusammenarbeit mit den deutschen Museumsämtern und Museumsverbänden auf Bundesebene gut vertraut, andererseits stand die Landesstelle für die nichtstaatlichen Museen in Bayern auch mit Kooperationspartnern im europäischen Ausland in enger Verbindung. Das war auch der Ausgangspunkt meines Interesses an der Erweiterung meiner Erfahrungen in der internationalen Museumsarbeit: Die Mitarbeit im Vorstand von ICOM Deutschland als der nationalen Vertretung des Weltmuseumsverbandes führte mich an die wichtigste Schnittstelle zwischen der nationalen und der internationalen Museumswelt, und genau da wollte ich hin.

\section{Aller Anfang ist schwer: Wie haben Sie Ihre ersten Gehversuche im Vorstand von ICOM Deutschland in Erinnerung?}

Wenn man aus München kommt, dann muss man sich schon erst einmal an die Berliner Luft gewöhnen. Und man muss auch seine eigene Rolle im Kreis der Kolleginnen und Kollegen im Vorstand suchen und seine besonderen beruflichen Erfahrungen einbringen. Unter dem Präsidenten Hans-Martin Hinz, in dessen beiden Amtsperioden von 1999 bis 2004 ich dem Vorstand angehörte, hatte sich ICOM Deutschland strukturell von einem ehrenamtlich geprägten Verein zu einem im öffentlichen kulturellen Leben wahrgenommenen Fachverband entwickelt. Hans-Martin brachte einschlägige Erfahrungen mit: Als Mitglied der Geschäftsleitung hatte er schon den Aufbau des Deutschen Historischen Museums maßgeblich mitbestimmt und mit seinen organisatorischen Fähigkeiten auch bei ICOM Deutschland eine dynamische Entwicklung eingeleitet.

Erst unter ihm hat ICOM Deutschland in schrittweisem Aufbau eine eigene Geschäftsstelle mit einer fest angestellten Geschäftsführerin, Frau Westphal, erhalten: Das war die notwendige organisatorische Grundlage für eine funktionierende Mitgliederbetreuung sowie für aktive Zusammenarbeit mit dem Weltverband von ICOM und seinen zahlreichen Fachkomitees. Diesen Aufschwung habe ich miterlebt, ebenso wie den Aufbau eines Netzwerks mit den Schwesterkomitees in den europäischen Nachbarländern, gerade in der Phase 
der Erweiterung der Möglichkeiten der Zusammenarbeit mit den osteuropäischen Nachbarländern nach der Grenzöffnung I989.

Hans-Martin Hinz setzte sich im Anschluss an erste Initiativen seines Vorgängers Hans-Albert Treff mit Erfolg für die Unterbringung der neuen Geschäftsstelle von ICOM Deutschland im Gebäude des »Instituts für Museumskunde« der Staatlichen Museen zu Berlin ein, wo auch die Geschäftsstelle des Deutschen Museumsbundes residierte. Die Aufnahme der Geschäftsstellen der beiden großen deutschen Museumsorganisationen unter das Dach des bundesweit tätigen Forschungs- und Dokumentationsinstituts unter seinem kooperativen und kulturpolitisch bestens vernetzten Leiter Prof. Bernhard Graf ließ in dem in Fachkreisen bekannten Haus In der Halde I in Berlin-Dahlem eine Drehscheibe der deutschen Museumspolitik entstehen, mit allen sich daraus ergebenden Möglichkeiten des Austauschs und der Zusammenarbeit.

Bei meiner Nachfolge auf Hans-Martin Hinz als Präsident von ICOM Deutschland zum Jahresbeginn 2005 konnte ich mich also auf ein kompetentes Sekretariat stützen. Ein geordneter Geschäftsgang und insbesondere auch die enge Kommunikation mit der Geschäftsstelle in Berlin als Rückgrat unserer Verbandsarbeit waren für mich bei der Wahrnehmung meiner Aufgaben an meinem entfernten Dienstort in München ausschlaggebend, auch wenn Dienstreisen nach Berlin immer wieder anstanden. In dankbarer Erinnerung möchte ich hier Johanna Westphal und ihr Team nennen: Johanna als hauptamtliche Geschäftsführerin hatte den »Laden im Griff « und bestimmte - gemeinsam mit den freiberuflichen Mitarbeiterinnen und Mitarbeitern - den »Auftritt« von ICOM Deutschland maßgeblich mit: Über die Amtszeit von vier Präsidenten war sie die Anlaufstelle für die ICOM-Mitglieder und primäre Ansprechpartnerin des Generalsekretariats in Paris, vor allem auch in den oft mühsamen Organisations- und Verwaltungsangelegenheiten: Das Abarbeiten der Themen Haushalt, Einzug und Verbuchung der Beiträge - von schon in meiner Zeit zuletzt etwa 4.000 Mitgliedern - unter Überweisung des Löwenanteils nach Paris, dazu Planung, Vorbereitung, Durchführung und Abrechnung von Veranstaltungen, Herausgabe der »ICOM Deutschland Mitteilungen«, das war der wenig sichtbare Teil der Aufgaben von ICOM Deutschland, für den es nur selten Anerkennung und schon gar keinen Applaus gab.

Was immer ICOM Deutschland in meiner Zeit erreichte, das erwuchs aus der guten Zusammenarbeit des Präsidenten mit dem Vorstand auf der Basis der Leistungen des Sekretariats einschließlich der Beiträge der freien Mitarbeiterinnen und Mitarbeitern. 
Die Wechselbeziehungen zwischen der nationalen und der internationalen Museumsarbeit haben bei Ihnen auch schon vor Ihrer Mitgliedschaft im Vorstand von ICOM Deutschland eine Rolle gespielt: Hat Ihr Engagement bei und für ICOM trotzdem eine Herausforderung für Sie bedeutet?

Wir haben eingangs schon darüber gesprochen, dass die Landesstelle für die nichtstaatlichen Museen in Bayern, als deren Leiter ich tätig war, schon immer sehr gut vernetzt war und in regem Austausch mit den Museumsorganisationen in den benachbarten Bundesländern stand, ebenso wie mit unseren europäischen Nachbarn. Mit der deutschen Wiedervereinigung I989 und der Durchlässigkeit der Grenzen zwischen West und Ost eröffneten sich neue Möglichkeiten für einen Austausch im Bereich der Museumsarbeit. Dabei ging es auch um die Aufarbeitung schwieriger Themen: Ein bayerisches Beispiel sind etwa die nach dem Kriege entstandenen Vertriebenenmuseen, die zunächst der Dokumentation der Kultur und des Lebens in der verlorenen Heimat gewidmet waren und sich erst allmählich auf einen offeneren Umgang mit der Geschichte der Volksgruppen und der Nationalitätenkonflikte mit ihren Folgen einließen, auch im grenzüberschreitenden Dialog bei gemeinsamen Fachtagungen. Insofern konnte ich mich bei meiner Mitarbeit im Vorstand von ICOM Deutschland auf Erfahrungen in der überregionalen und in der grenzüberschreitenden Museumsarbeit stützen, doch eröffnete sich über das Netzwerk von ICOM noch einmal eine ganz andere Dimension mit einem weltweiten Blick auf andere Gesellschaften und Kulturen sowie auf und die jeweilige Stellung der Museen im gesellschaftlichen Dialog.

Vom Beginn meiner Mitarbeit im Vorstand im Jahre I999 an und sehr viel intensiver mit der Übernahme des Präsidentenamts 2005 nahmen mich der Aufbau und die Pflege von Kontakten mit Partnern und Partnerverbänden im In- und Ausland in Anspruch. Ich denke dabei vor allem auch an die häufigen Dienstreisen, beispielsweise bei der Vorbereitung und Durchführung gemeinsamer Tagungen mit ICOM-Nationalkomitees in Partnerländern oder bei den jährlichen Sitzungen des Advisory Committee - also der den Vorstand des Weltverbandes beratenden Vertretung der Nationalkomitees - am Sitz von ICOM International in Paris. So bereichernd und verbindend diese Reisen waren, so waren sie jedenfalls keine Vergnügungsreisen, denn sie verlangten jedes Mal wieder eine Vorbereitung und das Absolvieren eines Programms mit Veranstaltungen, Begegnungen und Gesprächen, meist mit Englisch als Konferenzsprache. Zur Frage, ob das Engagement bei und für ICOM eine Herausforderung für mich bedeutet hat, lässt sich zusammenfassend sagen: Diesen Parcours zu bewältigen war schon nicht ganz einfach, auch wegen des Zeitdrucks, denn die Tätigkeit für ICOM als Ehrenamt musste mit meinen eigentlichen hauptberuflichen Aufgaben in Einklang gebracht werden. Im 
Hinblick auf die absehbaren Überschneidungen hatte ich beim Bayerischen Staatsministerium für Wissenschaft Forschung und Kunst vor meiner Kandidatur die Genehmigung eingeholt, im erforderlichen Umfang von meinen regelmäßigen Dienstzeiten freigestellt zu werden, um die als Präsident von ICOM Deutschland anfallenden Aufgaben wahrnehmen zu können. Doch trotz dieser Doppelbelastung fühle ich mich auch im Rückblick durch die zahlreichen Begegnungen und Erlebnisse belohnt, die doch in gewisser Weise Brücken gebaut haben. Gerne habe ich meine Möglichkeiten genutzt, im Rahmen unserer Auslandskontakte ein aktuelles Bild des gesellschaftlichen Lebens und der Arbeit der Museen in Deutschland zu vermitteln, gerade vor dem Hintergrund der noch lebendigen Erinnerungen an die beiden Weltkriege und die Zeit des Nationalsozialismus. Umgekehrt hat es den Blick geweitet, das Selbstverständnis der Museen und die Wahrnehmung ihres Bildungsauftrags in anderen Ländern und Gesellschaften kennen zu lernen.

\section{Um den Fokus nun mehr auf die nationale Museumsarbeit zu legen: Gab es von Deutschland - also von innen heraus - Themen, die aufgegriffen werden mussten?}

Traditionell standen in den deutschen Museen die Aufgaben des Sammelns und Bewahrens im Vordergrund. Dabei waren die Kriterien des Sammelns maßgeblich bestimmt vom materiellen Wert, der Seltenheit, der künstlerischen oder kunstvollen Gestaltung der Objekte, dazu von der historischen Bedeutung: Der Begriff der »Schausammlung « reflektiert noch etwas von den Wurzeln der Museen in den Galerien und Wunderkammern als ihren Vorläufern. Schon seit der »Kulturrevolution« der I968er Jahre wurde der gesamtgesellschaftliche Auftrag der Museen thematisiert: Was können die Museen beitragen zur gesellschaftlichen Entwicklung als Orte der Bildung und Information, nicht nur für das klassische Museumspublikum? Damit trat neben die Museumsaufgabe des »Ausstellens « verstärkt der Auftrag des »Vermittelns«: Die damals kontrovers geführte Diskussion über das besucherorientierte Museum befasste sich nun mit den Bezügen zwischen den Objekten und den Museumsbesuchern aus den unterschiedlichen sozialen Gruppen und auch Randgruppen bis hin zu den Nichtbesuchern. Das Thema scheint nach wie vor aktuell, und die bei der 25. ICOM-Generalkonferenz von Kyoto geführte Diskussion über eine Neufassung der weltweit als Richtlinie der Museumsarbeit anerkannten ICOM-Museumsdefinition unter Einbeziehung gesellschaftspolitischer Zielsetzungen ist nicht abgeschlossen.

Der Auftrag der Museen und die Gewichtung der Aufgaben des Bewahrens und Erhaltens sowie des Forschen und Vermittelns hat auch in meinen Jahren im Vorstand von ICOM die fachliche Diskussion bestimmt und eine Positio- 
nierung der Fachverbände veranlasst. Ausgehend von den Kernaussagen der ICOM-Museumsdefinition hat der Deutsche Museumsbund in Zusammenarbeit mit ICOM Deutschland 2006 die »Standards für Museen« als weiterführende Handreichung für die Museumsarbeit in Deutschland herausgegeben. Maßgeblicher Beweger dieses Projekts war Hans Lochmann, Mitglied des DMB-Vorstands und Geschäftsführer des Museumsverbandes Niedersachsen und Bremen, unterstützt von Michael Eissenhauer, damals Präsident des Deutschen Museumsbundes und gleichzeitig Mitglied des Vorstands von ICOM Deutschland. Die gemeinsame Erarbeitung der Standards für Museen war ein wichtiges Projekt in meiner Amtszeit und auch ein Prüfstein der guten Zusammenarbeit zwischen dem DMB und ICOM Deutschland. Es war mir stets ein Anliegen, die Zusammenarbeit der beiden großen deutschen Museumsverbände zu harmonisieren und in ihrer Ausrichtung zu definieren. Vereinfachend gesagt hat sich ICOM Deutschland schwerpunktmäßig um die »Außenpolitik « und den grenzüberschreitenden Wissenstransfer gekümmert, der DMB um die »Innenpolitik« und die Vertretung der Interessen der Museen und der Museumsmitarbeiterinnnen und -mitarbeiter.

\section{Welche Themen waren in Ihrer Amtszeit aktuell und haben Sie besonders be- schäftigt?}

Beim Blick zurück nehme ich wahr, dass manche heute aktuelle Themen schon in meiner Amtszeit gesellschaftliche und museumsfachliche Diskussionen ausgelöst haben. ICOM Deutschland hat sich diesen Fragen gestellt und auch heiße Eisen aufgegriffen: Gerade wenn es um grenzübergreifende Probleme ging, sah sich ICOM Deutschland als das zuständige Forum, um diese $\mathrm{zu}$ analysieren und $\mathrm{zu}$ reflektieren.

Manche der aktuellen Themen standen auch in Zusammenhang mit der Veranstaltung der Internationalen Museumstage jener Jahre, die von ICOM Deutschland in Zusammenarbeit mit dem Deutschen Museumsbund und in Partnerschaft mit der Sparkassen-Finanzgruppe ausgerichtet wurden. So wurde etwa das Motto des Museumstages 2005 »Museen bauen Brücken«/ «Museums bridging Cultures« in einer gemeinsam mit ICOM UK im Herbst 2005 in London realisierten Jahrestagung vertieft. Dieses Unternehmen diente - dem Selbstverständnis von ICOM entsprechend - in mehrfachem Sinne einem Brückenbau. Es ging darum, unseren deutschen Mitgliedern Kontakte und Einsichten in englische Museumsarbeit vor Ort zu vermitteln, gerade weil die englischen Museen eine beispielgebende Vorreiterrolle im Bereich der Besucherorientierung spielen. Umgekehrt war es ein Anliegen, eine Vorstellung vom aktuellen Selbstverständnis der deutschen Museen zu vermitteln, nachdem in England die Schatten der Nazizeit nach wie vor präsent 
sind. Schließlich ging es auch um die Erfahrungen in England als einem Einwanderungsland, wie Minderheiten mit Museumskonzepten und spezialisierten Angeboten angesprochen werden können. Solche Erfahrungen sind heute auch bei uns selbst gefragt, nachdem sich inzwischen auch in Deutschland die Frage stellt, welchen Beitrag die Museen zur Integration von Zuwanderern aus anderen Kulturkreisen leisten können.

Der Themenkreis des interkulturellen Dialogs wurde erneut aufgegriffen in der im Herbst 2008 ausgerichteten Jahrestagung »Museen - Orte der kulturellen Bildung und Integration «, diesmal realisiert als Gemeinschaftsveranstaltung mit ICOM NL in der Reinwardt-Akademie in Amsterdam mit maßgeblicher Unterstützung von Léontine Meijer van Mensch, damals Dozentin an der Amsterdamer Hochschule der Künste. Es ging um das Spannungsverhältnis von Identität und Integration und um die Rolle, welche die Museen als Lernund Erlebnisorte spielen können. Seit 2008 hat sich viel getan: Die Tagung in Amsterdam war ein Pilotprojekt, heute sind Migration und Integration als gesellschaftliche Herausforderung in der besucherorientierten Museumsarbeit verankert, mit der Akzentverschiebung von Integration zur Inklusion.

Auf Ihre Frage nach den in meiner Amtszeit aktuellen Themen möchte ich als einen weiteren Schwerpunkt den Kulturgutschutz herausgreifen, mit dem sich ICOM Deutschland eigentlich durchgehend befasst hat. Die Rückgabe von Kulturgütern, die im Nationalsozialismus beschlagnahmt bzw. nach der deutschen Jurisdiktion »verfolgungsbedingt entzogen« wurden, vor allem im Rahmen der Arisierung jüdischen Vermögens und Kunstbesitzes, ist im Zusammenhang mit dem Io. Jahrestag der »Washingtoner Erklärung« im Dezember 2008 erneut zu einem brisanten kulturpolitischen Thema geworden. Die Umsetzung der so genannten »Washingtoner Prinzipien« durch die »Gemeinsame Erklärung der Bundesregierung, der Länder und der kommunalen Spitzenverbände zur Auffindung und Rückgabe NS-verfolgungsbedingt entzogenem Kulturgut«, insbesondere durch Recherchen in den Sammlungsbeständen der Museen in öffentlich-rechtlicher Trägerschaft und die Rückgabe belasteter Objekte war zunächst schwer angelaufen. ICOM Deutschland war in die dadurch in Gang gekommene Fachdiskussion einbezogen und entsandte den Präsidenten in den wissenschaftlichen Beirat der 1998 in Magdeburg eingerichteten Koordinierungsstelle für Kulturgutverluste, die als Grundlage für die Dokumentation und Recherchen verfolgungsbedingt entzogenen Kulturguts sowie für die Einleitung von Restitutionsverfahren die Datenbank lostart. de aufbaute. Grundlageninformationen dazu wurden auch durch die Berichterstattung in den ICOM Deutschland Mitteilungen an die Mitglieder vermittelt. 
Ein anderer Aspekt des Kulturgutschutzes, der uns damals beschäftigte, war die komplexe Materie des »illicit traffic«, also des unerlaubten Handels mit Kulturgütern, die unter Verstoß gegen Gesetze oder die Ethischen Richtlinien in Verkehr gebracht werden. In meiner Amtszeit hatte der Einsatz von ICOM gegen den unerlaubten Handel mit Kulturgütern gerade auch in Deutschland gesteigerte Bedeutung, trotz des lediglich appellativen Charakters, nachdem das 2016 in Kraft getretene Kulturgutschutzgesetz noch in weiter Ferne lag. Dabei ging es auch darum, im Handel die Bereitschaft $z u$ wecken, zunächst einmal die eigenen Richtlinien des Kunsthandelsverbandes zu beachten, darüber hinaus aber auch die Handelsgepflogenheiten bei der Einfuhr, beim Erwerb und beim Verkauf insbesondere von antiken und außereuropäischen Kulturgütern verschärften Maßstäben zu unterwerfen und insbesondere auch Herkunftsnachweise zur legalen Provenienz der Objekte zu führen. Ein Instrument waren dabei die sogenannten »Roten Listen« von ICOM, in welchen Objekte, die aus illegalen Ausgrabungen stammen oder bei Diebstählen und Plünderungen entwendet wurden, dokumentiert werden. Ich erinnere mich noch an eine Pressekonferenz im Staatlichen Museum für Völkerkunde heute »Museum Fünf Kontinente - in München im Januar 2009, in deren Rahmen die von ICOM herausgegebene »Rote Liste der Antiken Perus « vorgestellt wurde. Adressaten sind nicht nur die Museen und der Kunsthandel, vielmehr unterstützen die »Roten Listen « auch die Zoll- und Polizeibehörden bei der Identifizierung widerrechtlich eingeführter Kulturgüter. Dementsprechend war die Pressekonferenz zur Präsentation der »Roten Liste Peru« ausgestaltet als ein kleines Symposium mit Beiträgen u. a. des peruanischen Botschafters, des Amerika-Referenten des Deutschen Archäologischen Instituts und eines Vertreters von Interpol, wie es dem Selbstverständnis von ICOM Deutschland als ein Forum für eine Zusammenarbeit im Netzwerk entspricht.

\section{Als Präsident von ICOM Deutschland haben Sie - bildlich gesprochen - einen Staffelstab übernommen und diesen weitergegeben: Wie sehen Sie die Ent- wicklung von ICOM Deutschland in ihrer Amtszeit?}

Bei Antritt meines Amtes vermochte ich mich auf eine funktionierende Struktur zu stützen, die maßgeblich von ehrenamtlichem Engagement getragen war, aber dank der schon eingangs erwähnten organisatorischen Aufbauarbeit meines Vorgängers Hans-Martin Hinz auch über eine zuverlässige, erfahrene und leistungsfähige Geschäftsstelle verfügte. Vielleicht muss man sich auch vergegenwärtigen, dass in der Zeit um die Jahrtausendwende die Entwicklung der EDV einen Quantensprung machte und der Computer als Basiswerkzeug in die Büros einzog. Damit eröffneten sich ganz neue Möglichkeiten für die Verwaltung - in unserem Fall der Mitgliederverwaltung und der Haushaltfüh- 
rung - wie auch für die EDV-gestützte Kommunikation: Das ermöglichte den Aufbau von Kontakten zu den Mitgliedern, die Verbreitung von Informationen und insbesondere die Einrichtung eines aktuellen Newsletters und den Versand von Einladungen und Tagungsunterlagen. Auch die Verbindung mit dem Generalsekretariat in Paris und zum Netzwerk des Weltverbandes mit seinen Gremien und den internationalen Fachkomitees wurde damit auf eine neue Grundlage gestellt. Das steigerte auch die Präsenz von ICOM Deutschland in der Öffentlichkeit und Fachöffentlichkeit und war damit sicher auch Motor der nach der Jahrtausendwende deutlich ansteigenden Mitgliederzahlen.

Die sich rasant entwickelnde Kommunikationstechnik war das Eine, »manpower « - im Fall unserer Geschäftsstelle vor allem »womanpower « - das Andere, denn mit den neuen Möglichkeiten wuchsen auch die Ansprüche und die Aufgaben. Dankbar erinnere ich mich an die weitgehend eigenverantwortliche, fachlich wie persönlich engagierte Aufbauarbeit der Geschäftsstelle mit ihren fest angestellten wie freiberuflichen Mitarbeiterinnen und Mitarbeiter. Wie unverzichtbar der laufende Einsatz und das Mitdenken Geschäftsstelle waren, merkte ich eigentlich nur, wenn ausnahmsweise einmal etwas nicht funktionierte.

Im Spektrum der inhaltlichen Verbandsarbeit bot die Fortsetzung und Vertiefung der Kooperationen mit den Nationalkomitees in europäischen Nachbarländern die Chance, im Vergleich der Lebensverhältnisse und der jeweiligen Konzepte der Museumsarbeit Erfahrungen auszutauschen und gesellschaftliche Fragen multiperspektivisch zu betrachten. Es fällt ja bekanntlich oft leichter, aus den Fehlern und Erfolgen der Nachbarn zu lernen, denn hier ist die notwendige Distanz gegeben, während man sich im eigenen Umfeld einer gewissen Betriebsblindheit oft nicht ganz entziehen kann. HansMartin Hinz als Präsident von ICOM Deutschland hatte es in seiner Amtszeit als eine besondere Kompetenz von ICOM Deutschland erkannt, die internationale Vernetzung unseres Verbandes auch bei der Veranstaltung gemeinsamer Tagungen ins Spiel zu bringen und durch persönliche Begegnungen zu vertiefen. Auch schon vor seiner Zeit war dieses Modell in kleinerem Format erprobt worden mit der Einführung der in dreijährigem Turnus seit I973 ausgerichteten trilateralen Bodenseetagungen als Gemeinschaftsveranstaltung der ICOM-Nationalkomitees der Bodensee-Anrainer Österreich, Schweiz, und Deutschland, seinerzeit initiiert von Prof. Hermann Auer, dem langjährigen Präsidenten von ICOM Deutschland (I968-I992). In meine Amtszeit fielen die I2. Bodenseetagung 2006, die vom Gastgeberland Schweiz in Schaffhausen unter dem Leitthema »Das Museum als Ort des Wissens« ausgerichtet wurde, sowie die I3. Bodenseetagung 2009 unter dem praxisorientierten Motto »Museen und Tourismus«, bei der ich das Präsidentenamt vorzeitig an 
meinen Nachfolger Klaus Weschenfelder übergab. Im Jahr nach dem Ende des aktiven Berufslebens fehlte mir vielleicht die bis dahin herrschende Wechselspannung, aus der sich auch die Wahrnehmung der ehrenamtlichen Aufgaben speiste. Doch kamen damals auch besondere gesundheitliche und persönliche Belastungen hinzu, die meine Rücktrittsentscheidung begründeten. Ich war und bin Klaus Weschenfelder, dem ich mich auch heute noch freundschaftlich verbunden fühle, dankbar für sein Verständnis und für seine Bereitschaft, auf halber Strecke Würde und Bürde des verantwortungsvollen Präsidentenamtes an der Spitze des deutschen Nationalkomitees von ICOM zu übernehmen.

Doch nach diesem persönlichen Exkurs möchte ich noch einmal zusammenfassend zurückkommen auf die Kooperationen mit ICOM Österreich, ICOM Schweiz, ICOM Frankreich, ICOM UK und ICOM Niederlande: Dieses Netzwerk hat in meiner Amtszeit eine wesentliche Rolle für die gemeinsame Bearbeitung von Schwerpunktthemen im Rahmen unseres Veranstaltungsprogramms gespielt. Aber auch bei der Vertretung europäischer Interessen im Rahmen der Sitzungen des Advisory Committee oder bei den Generalkonferenzen kam man in diesem vertrauten Kreis zusammen: Bei diesen über die Jahre entstandenen Kontakten verband sich der kollegiale Austausch mit den freundschaftlichen Begegnungen und Wiederbegegnungen, - so bei der in meine Amtszeit fallenden 2I. Generalkonferenz in Wien, die sich unter dem Motto »Museen und universelles Erbe « mit der Rolle der Museen in einer globalisierten und zunehmend virtuellen Welt auseinandersetzte.

\section{Wie denken Sie, sollte ICOM Deutschland heute mit den aktuellen Herausfor- derungen umgehen?}

Der Auftrag und die Tätigkeit von ICOM Deutschland leitet sich ab aus dem Status als deutsches Nationalkomitee des Weltverbandes. Hieraus ergibt sich dessen Stellung als international ausgerichteter Fachverband, wie sie auch in der Satzung definiert wird. Wie die Gründung der UNO und der UNESCO fällt die Errichtung von ICOM in die Zeit unmittelbar nach dem Ende des Zweiten Weltkrieges. Bei der Entstehung des Internationalen Museumsrats klingen die Kriegsereignisse nach in dem Anliegen, sich für die Pflege und Erhaltung der Kulturgüter, und der Museen, die sie erhalten und bewahren weltweit einzusetzen und Standards für Museen und ihre Sammlungen sowie für die professionellen Aufgaben der Museumsmitarbeiter Standards zu formulieren. In den seither vergangenen sieben Jahrzehnten hat sich ICOM zu einer anerkannten Museumsorganisation mit einer differenzierten Verwaltungsstruktur und derzeit II9 eigenständigen Nationalkomitees und 32 Fachkomitees entwickelt. Die Stellungnahmen von ICOM zu kultur- und museumspolitischen Themen finden weithin Beachtung. Allerdings scheinen mir persönlich die 
Verfestigung der tradierten Verbandsstruktur und ihre Komplexität auch eine gewisse Unbeweglichkeit mit sich zu bringen. Das gilt gerade in Zeiten, in denen eine profilierte und aktive Präsidentenfigur an der Spitze fehlt: Ich denke an die krisenhafte Phase vor der Bestellung des derzeit amtierenden Präsidenten im vergangenen Jahr. In meiner Amtszeit hat Alissandra Cummins als dynamische Präsidentin ICOM Impulse und ein Gesicht gegeben, ebenso HansMartin Hinz als ihr Nachfolger, der mit seinen organisatorischen Fähigkeiten, seinen diplomatischen Umgangsformen und seinem ergebnisorientierten Handeln den Weltverband so erfolgreich leitete.

Vor diesem Hintergrund trat die im letzten Jahr zu beobachtende Führungsschwäche, die mit der mangelnden Kontrolle und Steuerung der aufgebrochenen Diskussion über eine programmatische Neufassung der über Jahrzehnte bewährten Museumsdefinition einherging, besonders deutlich hervor. Das in der derzeit noch verbindlichen ICOM-Museumsdefinition verankerte Kriterium, dass Museen mit ihren Sammlungen im Dienst der Gesellschaft und ihrer Entwicklung stehen, sagt jedenfalls mehr aus und ist zugleich wandlungsfähiger und offener, als der von einem damit beauftragten internationalen ICOM-Komitee »Museum Definition, Prospects and Potentials « (MDPP) Entwurf für eine programmatische Neufassung. Schon der erste Satz dieses sozial-lyrischen Mission Statements »Museen sind demokratisierende, inklusive und polyphone Räume für kritischen Dialog über die Vergangenheit und Zukunft « macht nachvollziehbar, warum der Entwurf von der ICOM-Generalversammlung am 7. September 2019 in Kyoto auf einer außerordentlichen Sitzung mit 70\% der Stimmen abgelehnt wurde.

Die Eingangsfrage, wie ICOM Deutschland mit den heutigen Herausforderungen umgehen soll, möchte ich vor allem auch auf diese aktuelle, noch nicht ausgestandene Debatte über eine neue Standortbestimmung beziehen: Ich möchte abschließend der Hoffnung Ausdruck verleihen, dass ICOM Deutschland mit seinem Gewicht als weltweit mitgliederstärkstes Nationalkomitee einen Beitrag dazu leisten kann, die Kontinuität der Arbeit des Weltverbandes abzusichern und diese auf der Basis seiner bewährten und weltweit anerkannten Grundsätze weiterzuführen. Soweit ich das als zurückgezogener Ruheständler über die Medien verfolgen kann, scheint sich der Vorstand von ICOM Deutschland engagiert und besonnen an dieser Grundsatzdiskussion zu beteiligen: Das gilt sowohl für die Präsidentin als auch für ihren Stellvertreter, denen ich bei dieser Gelegenheit für ihren weiteren Einsatz Erfolg wünschen möchte. 


\subsection{Kommentierung durch die Interviewerin}

Das Interview mit Dr. York Langenstein fand in seinem Privathaus in München statt. Nach kleinen Startschwierigkeiten bezüglich der Terminfindung und anschließenden Unsicherheiten wegen der gerade startenden Corona-Pandemie gestaltete sich das Gespräch offen und unproblematisch. Dr. Langenstein hatte bereits ein Dokument vorbereitet, in dem er die ersten Fragen des ICOMFragenkataloges schriftlich beantwortete. Da dies aber nicht gewählter Forschungsweise entsprach, starteten wir selbstverständlich erneut und von Beginn an mit dem kompletten Fragenkatalog, den er gerne und höchst eloquent beantwortete.

Die Gesprächssituation war stets entspannt, wenn auch leider öfter von Telefonanrufen unterbrochen, die einen Einschnitt in der Narrative bedeuteten und meist das Thema, über das gerade gesprochen wurde fallengelassen wurde und der logische Anschluss trotz Notizen meinerseits etwas verloren ging. Andere Ansätze wurden jedoch schnell aufgegriffen, sodass niemals eine unangenehme, stille Situation entstand. Dr. Langenstein war stets überaus höflich und wirkte trotz seines bewundernswerten Werdegangs und Lebenslaufes durchaus bescheiden. Diese Bescheidenheit drückte er stets im Hinblick auf seinen revolutionären Vorgänger, Prof. Dr. Hans-Martin Hinz aus, mit dem er nach seiner Aussage neben beruflichen und verbandstechnischen Aktivitäten auch eine private Freundschaft pflegt.

Merklich behutsam und vorsichtig drückte er sich bei möglicher Kritik an ICOMs Verbandsarbeit aus. Die ohnehin sehr wohlwollend formulierten und allem Anschein nach durchaus berechtigten Punkte betreffend die fachliche Unterstützung der Vorstandsebene, die er ansprach, wollte er vor Veröffentlichung unbedingt noch einmal mit Prof. Hinz abklären, wohl um ungewollten Aufruhr zu vermeiden. Dadurch entstand zuletzt eine etwas langwierige Überarbeitung seitens Dr. Langenstein. Das Endresultat weicht nicht nur vom ausgehenden Interview ab, sondern auch teils in den Eingangsfragen von gewähltem, vorgegebenen Leitfaden.

In der Gesamtheit war auch dieses Interview inklusive Gesprächssituation außerordentlich informativ. Leider kam es in der anschließenden Zusammenarbeit zu starken Verzögerungen. Letztendlich führte dies zu einem Endresultat, welches für die gewählte Forschungsmethode eher weniger zufriedenstellend war, da es von mehreren Personen gegengelesen, ergänzt und korrigiert wurde. 